Vladimir Vasilyev*

\title{
Elliptic operators and their symbols
}

https://doi.org/10.1515/dema-2019-0025

Received January 19, 2019; accepted July 19, 2019

Abstract: We consider special elliptic operators in functional spaces on manifolds with a boundary which has some singular points. Such an operator can be represented by a sum of operators, and for a Fredholm property of an initial operator one needs a Fredholm property for each operator from this sum.

Keywords: elliptic operator, local representative, enveloping operator

MSC: 47A05; 58J05

\section{Introduction}

This paper is devoted to describing the structure of a special class of linear bounded operators on a manifold with non-smooth boundary. Our description is based on Simonenko's theory of envelopes [1] and explains why we obtain distinct theories for pseudo-differential equations and boundary value problems and distinct index theorems for such operators.

\subsection{Operators of a local type}

In this section we will give some preliminary ideas and definitions from [1].

Let $B_{1}, B_{2}$ be Banach spaces consisting of functions defined on compact $m$-dimensional manifold $M$, $A: B_{1} \rightarrow B_{2}$ be a linear bounded operator, $W \subset M$, and $P_{W}$ be a projector on $W$, i.e.

$$
\left(P_{W} u\right)(x)= \begin{cases}u(x), & \text { if } x \in W \\ 0, & \text { if } x \notin \bar{W}\end{cases}
$$

Definition 1. An operator A is called an operator of local type if the operator

$$
P_{U} A P_{V}
$$

is a compact operator for arbitrary non-intersecting compact sets $U, V \subset M$.

\subsection{Simple examples}

These are two of the simplest examples for illustration.

Example 1. If $A$ is a differential operator of the type

$$
(A u)(x)=\sum_{|k|=0}^{n} a_{k}(x) D^{k} u(x), \quad D^{k} u=\frac{\partial^{k} u}{\partial x_{1}^{k_{1}} \cdots \partial x_{m}^{k_{m}}},
$$

then $A$ is an operator of local type.

*Corresponding Author: Vladimir Vasilyev: Chair of Differential Equations, Belgorod State National Research University, ul. Podedy 85, Belgorod 308015, Russia; E-mail: vladimir.b.vasilyev@gmail.com 
Example 2. If $A$ is a Calderon-Zygmund operator with variable kernel $K(x, y) \in C^{1}\left(\mathbb{R}^{m} \times\left(\mathbb{R}^{m} \backslash\{0\}\right)\right.$ of the following type

$$
(A u)(x)=v \cdot p \cdot \int_{\mathbb{R}^{m}} K(x, x-y) u(y) d y,
$$

then $A$ is an operator of a local type.

\section{Everywhere below we say "an operator" instead of "an operator of local type".}

\subsection{Functional spaces on a manifold}

\subsubsection{Spaces $H^{s}\left(\mathbb{R}^{m}\right), L_{p}\left(\mathbb{R}^{m}\right), C^{\alpha}\left(\mathbb{R}^{m}\right)$}

It is possible to work with distinct functional spaces $[2,3]$.

Definition 2. [4] The space $H^{s}\left(\mathbb{R}^{m}\right), s \in \mathbb{R}$, is a Hilbert space of functions with the finite norm

$$
\|u\|_{s}=\left(\int_{\mathbb{R}^{m}}|\widetilde{u}(\xi)|^{2}(1+|\xi|)^{2 s} d \xi\right)^{1 / 2},
$$

where the sign $\sim$ over a function means its Fourier transform.

Definition 3. [2] The space $L_{p}\left(\mathbb{R}^{m}\right), 1<p<+\infty$, is a Banach space of measurable functions with the finite norm

$$
\|u\|_{p}=\left(\int_{\mathbb{R}^{m}}|u(x)|^{p} d x\right)^{1 / p} .
$$

Definition 4. [2] The space $C^{\alpha}\left(\mathbb{R}^{m}\right), 0<\alpha \leq 1$, is a space of continuous functions $u$ on $\mathbb{R}^{m}$ satisfying the Hölder condition

$$
|u(x)-u(y)| \leq c|x-y|^{\alpha}, \quad \forall x, y \in \mathbb{R}^{m},
$$

with the finite norm

$$
\|u\|_{\alpha}=\inf \{c\},
$$

where infimum is taken over all constants $c$ from the above inequality.

\subsubsection{Partition of unity and spaces $H^{s}(M), L_{p}(M), C^{\alpha}(M)$}

If $M$ is a compact manifold then there is a partition of unity [5]. It means the following. For every finite open covering $\left\{U_{j}\right\}_{j=1}^{k}$ of the manifold $M$ there exists a system of functions $\left\{\varphi_{j}(x)\right\}_{j=1}^{k}, \varphi_{j}(x) \in C^{\infty}(M)$, such that

$$
\begin{aligned}
& -0 \leq \varphi_{j}(x) \leq 1, \\
& -\operatorname{supp} \varphi_{j} \subset U_{j}, \\
& -\sum_{j=1}^{k} \varphi_{j}(x)=1 .
\end{aligned}
$$

So we have

$$
f(x)=\sum_{j=1}^{k} \varphi_{j}(x) f(x)
$$

for an arbitrary function $f$ defined on $M$.

Since every set $U_{j}$ is diffeomorphic to an open set $D_{j} \subset \mathbb{R}^{m}$ we have corresponding diffeomorphisms $\omega_{j}: U_{j} \rightarrow D_{j}$. Further, for a function $f$ defined on $M$ we compose mappings $f_{j}=f \cdot \varphi_{j}$ and as long as 
supp $f_{j} \subset U_{j}$ we put $\hat{f}_{j}=f_{j} \circ \omega_{j}^{-1}$ so that $\hat{f}_{j}: D_{j} \rightarrow \mathbb{R}$ is a function defined on a domain of $m$-dimensional space $\mathbb{R}^{m}$. We can consider, for example, the following functional spaces [2-4].

Definition 5. A function $f \in H^{s}(M)$ if the following norm

$$
\|f\|_{H^{s}(M)}=\sum_{j=1}^{k}\left\|\hat{f}_{j}\right\|_{s}
$$

is finite.

A function $f \in L_{p}(M)$ if the following norm

$$
\|f\|_{L_{p}(M)}=\sum_{j=1}^{k}\left\|\hat{f}_{j}\right\|_{p}
$$

is finite.

A function $f \in C^{\alpha}(M)$ if the following norm

$$
\|f\|_{C^{\alpha}(M)}=\sum_{j=1}^{k} \mid \hat{f}_{j} \|_{\alpha}
$$

is finite.

\section{Operators on a compact manifold}

On the manifold $M$ we fix a finite open covering and a partition of unity corresponding to this covering $\left\{U_{j}, f_{j}\right\}_{j=1}^{n}$. We then choose smooth functions $\left\{g_{j}\right\}_{j=1}^{n}$ so that supp $g_{j} \subset V_{j}, \overline{U_{j}} \subset V_{j}$, and $g_{j}(x) \equiv 1$ for $x \in \operatorname{supp} f_{j}$, supp $f_{j} \cap\left(1-g_{j}\right)=\emptyset$.

Proposition 1. The operator $A$ on the manifold $M$ can be represented in the form

$$
A=\sum_{j=1}^{n} f_{j} \cdot A \cdot g_{j}+T
$$

where $T: B_{1} \rightarrow B_{2}$ is a compact operator.

Proof. The proof is straightforward. Since

$$
\sum_{j=1}^{n} f_{j}(x) \equiv 1, \quad \forall x \in M,
$$

then we have

$$
A=\sum_{j=1}^{n} f_{j} \cdot A=\sum_{j=1}^{n} f_{j} \cdot A \cdot g_{j}+\sum_{j=1}^{n} f_{j} \cdot A \cdot\left(1-g_{j}\right),
$$

and the proof is completed.

Remark 1. Obviously such an operator is defined uniquely up to a compact operators which have no influence on an index.

By definition, for an arbitrary operator $A: B_{1} \rightarrow B_{2}$

$$
\||| A \mid\| \equiv \inf \|A+T\|,
$$

where infimum is taken over all compact operators $T: B_{1} \rightarrow B_{2}$.

Let $B_{1}^{\prime}, B_{2}^{\prime}$ be Banach spaces consisting of functions defined on $\mathbb{R}^{m}$, and let $\widetilde{A}: B_{1}^{\prime} \rightarrow B_{2}^{\prime}$ be a linear bounded operator. 
Since $M$ is a compact manifold, then for every point $x \in M$ there exists a neighborhood $U \ni x$ and a diffeomorphism $\omega: U \rightarrow D \subset \mathbb{R}^{m}, \omega(x) \equiv y$. We denote by $S_{\omega}$ the following operator acting from $B_{k}$ to $B_{k}^{\prime}, k=1,2$. For every function $u \in B_{k}$ vanishing out of $U$

$$
\left(S_{\omega} u\right)(y)=u\left(\omega^{-1}(y)\right), \quad y \in D, \quad\left(S_{\omega} u\right)(y)=0, \quad y \notin D .
$$

Definition 6. A local representative of the operator $A: B_{1} \rightarrow B_{2}$ at the point $x \in M$ is called the operator $\widetilde{A}: B_{1}^{\prime} \rightarrow B_{2}^{\prime}$ such that for all $\varepsilon>0$ there exists the neighborhood $U_{j}$ of the point $x \in U_{j} \subset M$ with the property

$$
\left\|\left|g_{j} A f_{j}-S_{\omega_{j}^{-1}} \hat{g}_{j} \tilde{A} \hat{f}_{j} S_{\omega_{j}}\right|\right\|<\varepsilon .
$$

\section{Algebra of symbols}

Definition 7. Symbol of an operator $A$ is called the family of its local representatives $\left\{A_{x}\right\}$ at each point $x \in \bar{M}$.

One can show like [1] this definition of an operator symbol conserves all properties of a symbolic calculus. Namely, up to compact summands we have the following:

- the product and the sum of two operators corresponds to the product and the sum of their local representatives;

- the adjoint operator corresponds to its adjoint local representative;

- a Fredholm property of an operator corresponds to a Fredholm property of its local representative.

\section{Operators with symbols. Examples of operators}

It seems not every operator has a symbol, and we give some examples for operators with symbols.

Example 3. Let $A$ be the differential operator from Example 1, and functions $a_{k}(x)$ be continuous functions on $\mathbb{R}^{m}$. Then its symbol is an operator family consisting of multiplication operators on the function

$$
\sum_{|k|=0}^{n} a_{k}(x) \xi^{k},
$$

where $\xi^{k}=\xi_{1}^{k_{1}} \ldots \xi_{m}^{k_{m}}$.

Example 4. Let $A$ be the Calderon-Zygmund operator from Example 2 and $\sigma(x, \xi)$ be its symbol in the sense of [2], then its symbol is an operator family consisting of multiplication operators on the function $\sigma(x, \xi)$.

The more important point is that the symbol of an operator is simpler than general operator, and it permits to verify its Fredholm properties. For the two above examples a Fredholm property of an operator symbol is equivalent to its invertibility.

\section{Stratification of manifolds and operators}

\subsection{Sub-manifolds}

The above definition of an operator on a manifold supposes that all neighborhoods $\left\{U_{j}\right\}$ have the same type. But even if a manifold has a smooth boundary then there are two types of neighborhoods related to a placement of neighborhood, namely inner neighborhoods and boundary ones. For an inner neighborhood $U$ such that $\bar{U} \subset \stackrel{\circ}{M}$ we have the diffeomorphism $\omega: U \rightarrow D$, where $D \in \mathbb{R}^{m}$ is an open set. For a boundary neighborhood such that $U \cap \partial M \neq \emptyset$ we have another diffeomorphism $\omega_{1}: U \rightarrow D \cap \mathbb{R}_{+}^{m}$, where

$$
\mathbb{R}_{+}^{m}=\left\{x \in \mathbb{R}^{m}: x=\left(x_{1}, \cdots, x_{m}\right), x_{m}>0\right\} .
$$


Maybe this boundary $\partial M$ has some singularities like conical points and wedges. The conical point at the boundary is such a point, for which its neighborhood is diffeomorphic to the cone

$$
C_{+}^{a}=\left\{x \in \mathbb{R}^{m}: x_{m}>a\left|x^{\prime}\right|, x^{\prime}=\left(x_{1}, \ldots, x_{m-1}\right), a>0\right\} .
$$

The wedge point of codimension $k, 1 \leq k \leq m-1$, is such a point for which its neighborhood is diffeomorphic to the set $\left\{x \in \mathbb{R}^{m}: x=\left(x^{\prime}, x^{\prime \prime}\right), x^{\prime \prime} \in \mathbb{R}^{m-k}, x^{\prime}=\left(x_{1}, \cdots, x_{m-k-1}\right), x_{m-k-1}>a\left|x^{\prime \prime \prime}\right|, x^{\prime \prime \prime}=\left(x_{1}, \cdots, x_{m-k-2}\right), a>\right.$ $0\}$. So if the manifold $M$ has such singularities we suppose that we can extract certain k-dimensional submanifolds, namely an ( $m-1)$-dimensional boundary $\partial M$, and $k$-dimensional wedges $M_{k}, k=0, \cdots, m-2$; $M_{0}$ are a collection of conical points.

\subsection{Enveloping operators}

If the family $\left\{A_{x}\right\}_{x \in M}$ is continuous in the operator topology, then according to Simonenko's theory there is an enveloping operator, i.e. such an operator $A$ for which every operator $A_{x}$ is the local representative for the operator $A$ in the point $x \in M$.

Example 5. If $\left\{A_{x}\right\}_{x \in M}$ consists of Calderon-Zygmund operators in $\mathbb{R}^{m}$ [2] with symbols $\sigma_{x}(\xi)$ parametrized by points $x \in M$ and this family smoothly depends on $x \in M$ then the Calderon-Zygmubd operator with variable kernel and symbol $\sigma(x, \xi)$ will be an enveloping operator for this family.

Example 6. If $\left\{A_{x}\right\}_{x \in M}$ consists of null operators then an enveloping operator is a compact operator [1].

Theorem 1. The operator A has a Fredholm property if and only if its all local representatives $\left\{A_{x}\right\}_{x \in M}$ have the same property.

This property was proved in [1], but we will give the proof (see Lemma 2) including some new constructions because it will be used below for a decomposition of the operator.

\subsection{Hierarchy of operators}

We will remind the reader here of the following definition and Fredholm criteria for operators [6].

Definition 8. Let $B_{1}, B_{2}$ be Banach spaces, and $A: B_{1} \rightarrow B_{2}$ be a linear bounded operator. The operator $R: B_{2} \rightarrow B_{1}$ is called a regularizer for the operator $A$ if the following properties

$$
R A=I_{1}+T_{1}, \quad A R=I_{2}+T_{2}
$$

hold, where $I_{k}: B_{k} \rightarrow B_{k}$ is an identity operator, $T_{k} ; B_{k} \rightarrow B_{k}$ is a compact operator, $k=1,2$.

Proposition 2. The operator $A: B_{1} \rightarrow B_{2}$ has a Fredholm property if and only if there exists a linear bounded regularizer $R: B_{2} \rightarrow B_{1}$.

Lemma 1. Let $f$ be a smooth function on the manifold $M, U \subset M$ be an open set, and supp $f \subset U$. Then the operator $f \cdot A-A \cdot f$ is a compact operator.

Proof. Let $g$ be a smooth function on $M$, supp $g \subset V \subset M$, moreover $\bar{U} \subset V, g(x) \equiv 1$ for $x \in \operatorname{supp} f$. Then we have

$$
\begin{aligned}
& f \cdot A=f \cdot A \cdot g+f \cdot A \cdot(1-g)=f \cdot A \cdot g+T_{1}, \\
& A \cdot f=g \cdot A \cdot f+(1-g) \cdot A \cdot f=g \cdot A \cdot f+T_{2},
\end{aligned}
$$

where $T_{1}, T_{2}$ are compact operators. Let us denote $g \cdot A \cdot g \equiv h$ and write

$$
f \cdot A \cdot g=f \cdot g \cdot A \cdot g=f \cdot h, \quad g \cdot A \cdot f=g \cdot A \cdot g \cdot f=h \cdot f,
$$

and we obtain the required property. 
Definition 9. The operator $A$ is called an elliptic operator if its operator symbol $\left\{A_{x}\right\}_{x \in M}$ consists of Fredholm operators.

Now we will show that each elliptic operator really has a Fredholm property. Our proof in general follows the book [1], but our constructions are more stratified and we need such constructions below.

Lemma 2. Let A be an elliptic operator. Then the operator A has a Fredholm property.

Proof. To obtain the proof we will construct the regularizer for the operator $A$. For this purpose we choose two coverings like Proposition 1 and write the operator $A$ in the form

$$
A=\sum_{j=1}^{n} f_{j} \cdot A \cdot g_{j}+T
$$

where $T$ is a compact operator. Without loss of generality we can assume that there are $n$ points $x_{k} \in U_{k} \subset$ $V_{k}, k=1,2, \ldots n$. Moreover, we can construct such coverings by balls in the following way. Let $\varepsilon>0$ be a small enough number. First, for every point $x \in M_{0}$ we take two balls $U_{x}, V_{x}$ with the center at $x$ of radius $\varepsilon$ and $2 \varepsilon$ and construct two open coverings for $M_{0}$ namely $\mathfrak{U}_{0}=\cup_{x \in M_{0}} U_{x}$ and $\mathfrak{V}_{0}=\cup_{x \in M_{0}} V_{x}$. Second, we consider the set $L_{1}=\bar{M} \backslash \mathfrak{V}_{0}$ and construct two coverings $\mathfrak{U}_{1}=\cup_{x \in L_{1} \cap M_{1}} U_{x}$ and $\mathfrak{V}_{1}=\cup_{x \in L_{1} \cap M_{1}} V_{x}$. Further, we introduce the set $L_{2}=\bar{M} \backslash\left(\mathfrak{V}_{0} \cup \mathfrak{V}_{1}\right)$ and two coverings $\mathfrak{U}_{2}=\cup_{x \in L_{2} \cap M_{2}} U_{x}$ and $\mathfrak{V}_{2}=\cup_{x \in L_{2} \cap M_{2}} V_{x}$. Continuing these actions we will come to the set $L_{m-1}=\bar{M} \backslash\left(\cup_{k=0}^{m-2} \mathfrak{U}_{k}\right)$ which consists of smoothness points of $\partial M$ and inner points of $M$. We then construct two covering $\mathfrak{U}_{m-1}=\cup_{x \in L_{m-1} \cap \partial M} U_{x}$ and $\mathfrak{V}_{m-1}=\cup_{x \in L_{m-1} \cap \partial M} V_{x}$. Finally, the set $L_{m}=\bar{M} \backslash\left(\cup_{k=0}^{m-1} \mathfrak{U}_{k}\right)$ consists of inner points of the manifold $M$ only. We finish this process by choosing the covering $\mathfrak{U}_{m}$ for the latter set $L_{m}$. So, the covering $\cup_{k=0}^{m} \mathfrak{U}_{k}$ will be a covering for the whole manifold $M$. According to the compactness property we can take into account that this covering is finite, and the centers of balls which cover $M_{k}$ are placed at $M_{k}$.

Now we will rewrite the formula (1) in the following way

$$
A=\sum_{k=0}^{m}\left(\sum_{j=1}^{n_{k}} f_{j k} \cdot A \cdot g_{j k}\right)+T,
$$

where the coverings and partitions of unity $\left\{f_{j k}\right\}$ and $\left\{g_{j k}\right\}$ are chosen as mentioned above. In other words the operator

$$
\sum_{j=1}^{n_{k}} f_{j k} \cdot A \cdot g_{j k}
$$

is related to some neighborhood of the sub-manifold $M_{k}$; this neighborhood is generated by covering the sub-manifold $M_{k}$ by balls with centers at points $x_{j k} \in M_{k}$. Since $A_{x_{j k}}$ is a local representative for the operator $A$ at point $x_{j k}$ we can rewrite the formula (2) as follows

$$
A=\sum_{k=0}^{m}\left(\sum_{j=1}^{n_{k}} f_{j k} \cdot A_{x_{j k}} \cdot g_{j k}\right)+T .
$$

Let us denote $S_{\omega_{j}^{-1}} \hat{g}_{j} \equiv \tilde{g}_{j} a n d \hat{f}_{j} S_{\omega_{j}} \equiv \tilde{f}_{j}$. Further, we can assert that the operator

$$
R=\sum_{k=0}^{m}\left(\sum_{j=1}^{n_{k}} g_{j k} \cdot A_{x_{j k}}^{-1} f_{j k}\right)
$$

will be the regularizer for the operator $A^{\prime}$; here $A_{x_{j k}}^{-1}$ is a regularizer for the operator $A_{x_{j k}}$.

Indeed,

$$
R A=\left(\sum_{k=0}^{m}\left(\sum_{j=1}^{n_{k}} g_{j k} A_{x_{j k}}^{-1} f_{j k}\right)\right) \cdot A=\sum_{k=0}^{m} \sum_{j=1}^{n_{k}} g_{j k} \cdot A_{x_{j k}}^{-1} \cdot\left(A-A_{x_{j k}}+A_{x_{j k}}\right) \cdot f_{j k}+T_{1}
$$




$$
\begin{aligned}
& =\sum_{k=0}^{m} \sum_{j=1}^{n_{k}} g_{j k} \cdot A_{x_{j k}}^{-1} \cdot\left(A-A_{x_{j k}}\right) \cdot f_{j k}+\sum_{k=0}^{m} \sum_{j=1}^{n_{k}} f_{j k}+T_{1}=I_{1}+T_{1}+\Theta_{1}, \\
\Theta_{1} & =\sum_{k=0}^{m} \sum_{j=1}^{n_{k}} g_{j k} \cdot A_{x_{j k}}^{-1} \cdot\left(A-A_{x_{j k}}\right) \cdot f_{j k},
\end{aligned}
$$

because $f_{j k} \cdot A_{x_{j k}}=A_{x_{j k}} \cdot f_{j k}+$ compact summand, and $f_{j k} \cdot g_{j k}=f_{j k}$, and

$$
\sum_{k=0}^{m} \sum_{j=1}^{n_{k}} f_{j k} \equiv 1
$$

as the partition of unity. The same property

$$
\begin{gathered}
A R=I_{2}+T_{2}+\Theta_{2}, \\
\Theta_{2}=\sum_{k=0}^{m} \sum_{j=1}^{n_{k}} g_{j k} \cdot\left(A-A_{x_{j k}}\right) \cdot A_{x_{j k}}^{-1} \cdot f_{j k},
\end{gathered}
$$

is verified analogously.

\section{Piece-wise continuous operator families}

Given an operator $A$ with the symbol $\left\{A_{x}\right\}_{x \in \bar{M}}$ which generates a few operators in dependence on a quantity of singular manifolds; we consider this situation in the following way. We will assume additionally some smoothness properties for the symbol $\left\{A_{x}\right\}_{x \in \bar{M}}$.

Theorem 2. If the symbol $\left\{A_{x}\right\}_{x \in \bar{M}}$ is a piece-wise continuous operator function then there are $m+1$ operators $A^{(k)}, k=0,1, \ldots, m$ such that the operator $A$ and the operator

$$
A^{\prime}=\sum_{k=0}^{m} A^{(k)}+T
$$

have the same symbols, where the operator $A^{(k)}$ is an enveloping operator for the family $\left\{A_{x}\right\}_{x \in \bar{M}_{k}}$, andT is a compact operator.

Proof. We will use the constructions from the proof of Lemma 2, namely the formula (3). We will extract the operator

$$
\sum_{j=1}^{n_{k}} f_{j k} \cdot A_{x_{j k}} \cdot g_{j k}
$$

which "serves" the sub-manifold $M_{k}$ and consider it in detail. This operator is related to neighborhoods $\left\{U_{j k}\right\}$ and the partition of unity $\left\{f_{j k}\right\}$. Really, $U_{j k}$ is the ball with the center at $x_{j k} \in M_{k}$ of radius $\varepsilon>0$, and therefore $f_{j k}, g_{j k}, n_{k}$ depend on $\varepsilon$.

According to Simonenko's ideas [1] we will construct the component $A^{(k)}$ in the following way. Let $\left\{\varepsilon_{n}\right\}_{n=1}^{\infty}$ be a sequence such that $\varepsilon_{n}>0$ for all $n \in \mathbb{N}$, $\lim _{n \rightarrow \infty} \varepsilon_{n}=0$. Given $\varepsilon_{n}$ we choose coverings $\left\{U_{j k}\right\}_{j=1}^{n_{k}}$ and $\left\{V_{j k}\right\}_{j=1}^{n_{k}}$ as above with partition of unity $\left\{f_{j k}\right\}$ and corresponding functions $\left\{g_{j k}\right\}$ such that

$$
\left\|f_{j k} \cdot\left(A_{x}-A_{x_{k k}}\right) \cdot g_{j k}\right\| \mid<\varepsilon_{n}, \quad \forall x \in V_{j k} ;
$$

we remind that $U_{j k}, V_{j k}$ are balls with centers at $x_{j k} \in \overline{M_{k}}$ of radius $\varepsilon$ and $2 \varepsilon$. This requirement is possible according to continuity of family $\left\{A_{x}\right\}$ on the sub-manifold $\overline{M_{k}}$. Now we will introduce such a constructed operator

$$
A_{n}=\sum_{j=1}^{n_{k}} f_{j k} \cdot A_{x_{j k}} \cdot g_{j k}
$$


and will show that the sequence $\left\{A_{n}\right\}$ is a Cauchy sequence with respect to a norm $\|\mid \cdot\|$. We have

$$
A_{l}=\sum_{i=1}^{l_{k}} F_{i k} \cdot A_{y_{i k}} \cdot G_{i k},
$$

where the operator $A_{l}$ is constructed for a given $\varepsilon_{l}$ with corresponding coverings $\left\{u_{i k}\right\}_{i=1}^{l_{k}}$ and $\left\{v_{i k}\right\}_{j=1}^{i_{k}}$ with partition of unity $\left\{F_{i k}\right\}$ and corresponding functions $\left\{G_{i k}\right\}$ so that

$$
\left\|F_{i k} \cdot\left(A_{x}-A_{y_{i k}}\right) \cdot G_{i k}\right\| \mid<\varepsilon_{l}, \quad \forall x \in v_{i k} ;
$$

here $u_{i k}, v_{i k}$ are balls with centers at $y_{i k} \in \overline{M_{k}}$ of radius $\tau$ and $2 \tau$.

We can write

$$
\begin{aligned}
A_{n} & =\sum_{j=1}^{n_{k}} f_{j k} \cdot A_{x_{j k}} \cdot g_{j k}=\sum_{i=1}^{l_{k}} F_{i k} \cdot \sum_{j=1}^{n_{k}} f_{j k} \cdot A_{x_{j k}} \cdot g_{j k} \\
& =\sum_{i=1}^{l_{k}} \sum_{j=1}^{n_{k}} F_{i k} \cdot f_{j k} \cdot A_{x_{j k}} \cdot g_{j k}=\sum_{i=1}^{l_{k}} \sum_{j=1}^{n_{k}} F_{i k} \cdot f_{j k} \cdot A_{x_{j k}} \cdot g_{j k} \cdot G_{k}+T_{1},
\end{aligned}
$$

and the same can be done for $A_{l}$

$$
\begin{aligned}
A_{l} & =\sum_{i=1}^{l_{k}} F_{i k} \cdot A_{y_{i k}} \cdot G_{i k}=\sum_{j=1}^{n_{k}} f_{j k} \cdot \sum_{i=1}^{l_{k}} F_{i k} \cdot A_{y_{i k}} \cdot G_{i k}-\sum_{j=1}^{n_{k}} \sum_{i=1}^{l_{k}} f_{j k} \cdot F_{i k} \cdot A_{y_{i k}} \cdot G_{i k} \\
& =\sum_{j=1}^{n_{k}} \sum_{i=1}^{l_{k}} f_{j k} \cdot F_{i k} \cdot A_{y_{i k}} \cdot G_{i k} \cdot g_{j k}+T_{2} .
\end{aligned}
$$

Let us consider the difference

$$
\left\|A_{n}-A_{l}\left|\left\|=|| \mid \sum_{j=1}^{n_{k}} \sum_{i=1}^{l_{k}} f_{j k} \cdot F_{i k} \cdot\left(A_{x_{j k}}-A_{y_{i k}}\right) \cdot G_{i k} \cdot g_{j k}\right\| .\right.\right.
$$

Obviously, summands with non-vanishing supplements to the formula (5) are those for which $U_{j k} \cap u_{i k} \neq$ $\emptyset$. A number of such neighborhoods are finite always for arbitrary finite coverings, hence we obtain

$$
\begin{aligned}
\left\|A_{n}-A_{l}\right\| & \leq \sum_{j=1}^{n_{k}} \sum_{i=1}^{l_{k}}\left\|f_{j k} \cdot F_{i k} \cdot\left(A_{x_{j k}}-A_{y_{i k}}\right) \cdot G_{i k} \cdot g_{j k}\right\| \\
& \leq \sum_{x \in U_{j k} \cap u_{i k} \neq \emptyset}\left\|f_{j k} \cdot F_{i k} \cdot\left(A_{x_{j k}}-A_{x}\right) \cdot G_{i k} \cdot g_{j k}\right\| \mid+\sum_{x \in U_{j k} \cap u_{i k} \neq \emptyset}\left\|f_{j k} \cdot F_{i k} \cdot\left(A_{x}-A_{y_{i k}}\right) \cdot G_{i k} \cdot g_{j k}\right\| \\
& \leq 2 K \max \left[\varepsilon_{n}, \varepsilon_{l}\right],
\end{aligned}
$$

where $K$ is a universal constant.

Thus, we have proved that the sequence $\left\{A_{n}\right\}$ is a Cauchy sequence, hence there exists $\lim _{n \rightarrow \infty} A_{n}=A^{(k)}$.

Corollary 1. The operator A has a Fredholm property if and only if all operators $A^{(k)}, k=0,1, \ldots, m$ have the same property.

Remark 2. The constructed operator $A^{\prime}$ generally speaking does not coincide with the initial operator $A$ because they act in different spaces. But for some cases they may be the same.

\section{Conclusion}

This paper is a general concept of my vision to the theory of pseudo-differential equations and boundary value problems on manifolds with a non-smooth boundary. The second part will be devoted to applying these abstract results to index theory for such operator families and then to concrete classes of pseudo-differential equations. 


\section{References}

[1] Simonenko I. B., Local Method in the Theory of Translation Invariant Operators and Their Envelopes, Rostov on Don, 2007 (in Russian)

[2] Mikhlin S. G., Prössdorf S., Singular Integral Operators, Akademie-Verlag, Berlin, 1986

[3] Tribel H. Theory of Function Spaces, I, II, Birkhäuser, Basel, 2000

[4] Eskin G., Boundary Value Problems for Elliptic Pseudodifferential Equations, Ams, Providence, 1981

[5] Munkres J., Analysis on Manifolds, CRC Press, Boca Raton, 2018

[6] Krein S. G., Linear Equations in Banach Spaces, Birkhäuser, Basel, 1982 